\title{
On the Compositional Checking of Validity
}

Glynn Winskel

DAIMI PB - 324

July 1990

COMPUTER SCIENCE DEPARTMENT AARHUS UNIVERSITY

Ny Munkegade, Building 540 DK-8000 Aarhus C, Denmark

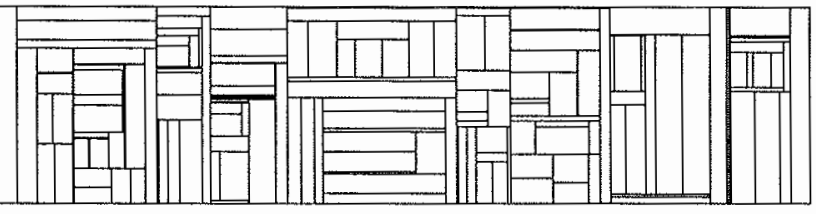




\section{ON THE COMPOSITIONAL CHECKING OF VALIDITY (Extended Abstract) \\ Glynn Winskel}

Computer Science Department, Aarhus University, Denmark

\section{Introduction}

This paper is concerned with deciding whether or not assertions are valid of a parallel process using methods which are directed by the way in which the process has been composed. The assertions are drawn from a modal logic with recursion, capable of expressing a great many properties of interest [EL]. The processes are described by a language inspired by Milner's CCS and Hoare's CSP, though with some modifications. The choice of constructors allows us to handle a range of synchronisation disciplines and ensures that the processes denoted are finite state. The operations are prefixing, a non-deterministic sum, product, restriction, relabelling and a looping construct. Arbitrary parallel compositions are obtained by using a combination of product, restriction and relabelling.

We are interested in deciding whether or not an assertion $A$ is valid of a process $t$. If it is valid, in the sense that every reachable state of $t$ satisfies $A$, we write $\models A: t$. Rather than perform the check $\models A: t$ monolithically, on the whole transition system denoted by the term $t$, we would often rather break the verification down into parts, guided by the composition of $t$. For instance if $t$ were a sum $t_{0}+t_{1}$ we can ask what assertions $A_{0}$ and $A_{1}$ should be valid of $t_{0}$ and $t_{1}$ respectively to ensure that $A$ is valid of $t_{0}+t_{1}$. This amounts to requiring assertions $A_{0}, A_{1}$ such that

$$
\models A: t_{0}+t_{1} \text { iff } \models A_{0}: t_{0} \text { and } \models A_{1}: t_{1} \text {. }
$$

Once the assertions $A_{0}$ and $A_{1}$ are found, a validity problem for $t_{0}+t_{1}$ is reduced to a problem to do with $t_{0}$ and another with $t_{1}$. Further, if the assertions can be found routinely only knowing the top-level operation, that e.g. the process is a sum, we are also told how to construct a process as a sum for which the assertion $A$ is valid: first find components $t_{0}$ and $t_{1}$ making $A_{0}$ and $A_{1}$ valid respectively. 
This paper investigates the extent to which the composition of $t$ can guide methods for deciding $\models A: t$. It formulates new compositional methods for deciding validity, and exposes some fundamental difficulties. Algorithms are provided to reduce validity problems for prefixing, sum, relabelling, restriction and looping to validity problems for their immediate components - all these reductions depend only on the top-level structure of terms. The existence of these reductions rests on being able to 'embed' the properties of a term in the properties, or products of properties, of its immediate subterms. Because there is not such a simple embedding for the product construction of terms, as might be expected, similar reductions become much more complicated for products; although there are general results, and the reductions can be simple in special cases, the general treatment for products meets with fundamental difficulties. Whereas reductions for products always exist for this finite state language, they demonstrably no longer just depend on the top-level (product structure) of the term; in particular, a simple assertion is exhibited for which the size of the reduction must be quadratic in the number of states of the process. An attempt is thus made to explain what makes product different from the other operations with respect to compositional reasoning, and to delimit the obstacles to automated compositional checking of validity on parallel processes.

\section{Transition systems and properties}

The syntax, presented formally in the next section, will consist of process terms and assertions.

Process terms will denote labelled transition systems with distinguished initial states. A labelled transition system is a structure $(S, i, L$, tran $)$ where $S$ is a set of states containing a distinguished state $i, L$ is a set of labels, and tran $\subseteq S \times L \times S$ is a set of transitions; as normal, we often write $s \stackrel{\alpha}{\longrightarrow} s^{\prime}$ if $\left(s, \alpha, s^{\prime}\right) \in$ tran. A state of a labelled transition system is reachable iff it can be obtained as the end state of a sequence of transition beginning at the initial state.

A closed assertion is to denote a property of a labelled transition system, i.e.a subset of its reachable states. We write $P(T)$ for the set of properties 
of a labelled transition system $T$.

We construct labelled transition systems using the constructions of prefixing, sum, product, restriction, relabelling and looping starting from the nil process. These operations form the basis of our syntax for processes. We now describe these constructions. As has been stated, properties of a labelled transition system are identified with subsets of reachable states. The constructions in our language of transition systems are associated with maps. These prove useful in importing properties of immediate components of a term into a property of the term itself. Such mappings between properties are a key to compositional reasoning about processes. We introduce them alongside the constructions with which they are associated.

nil: The nil transition system is $(\{i\}, i, \emptyset, \emptyset)$.

Prefixing: For a label $\alpha$ and a labelled transition system $T=(S, i, L$, tran $)$ the prefix $\alpha T$ is obtained by adjoining a new initial state and introducing an $\alpha$-transition from it to the old initial state. More concretely:

$$
\alpha T=\left(S^{\prime}, \emptyset, L \cup\{\alpha\}, \operatorname{tran}^{\prime}\right)
$$

where $S^{\prime}=\{\{s\} \mid s \in S\} \cup\{\emptyset\}$, and

$$
\begin{aligned}
\left(s_{1}, \beta, s_{1}^{\prime}\right) \in \operatorname{tran}^{\prime} \text { iff } & \left(s_{1}=\emptyset \& \beta=\alpha \& s_{1}^{\prime}=\{i\}\right) \text { or } \\
& \left(s_{1}=\{s\} \& s_{1}^{\prime}=\left\{s^{\prime}\right\} \&\left(s, \beta, s^{\prime}\right) \in\right. \text { tran } \\
& \text { for some } s, s^{\prime}
\end{aligned}
$$

There is map $S \rightarrow S^{\prime}$ taking $s \in S$ to the corresponding state $\{s\} \in S^{\prime}$. It extends to a map on properties $P(T) \rightarrow P(\alpha T)$. It is convenient to name this map on properties after the prefixing operation and we define

$$
\alpha(-): P(T) \rightarrow P(\alpha T)
$$

by taking $\alpha U=\{\{s\} \mid s \in U\}$ for $U \in P(T)$.

Sum: Let $T_{0}=\left(S_{0}, i_{0}, L_{0}, \operatorname{tran}_{0}\right)$ and $T_{1}=\left(S_{1}, i_{1}, L_{1}, \operatorname{tran}_{1}\right)$ be labelled transition systems. Our nondeterministic sum operation $T_{0}+T_{1}$ is a little different from Milner's. It identifies disjoint copies of the transition systems at their initial states. We define

$$
T_{0}+T_{1}=\left(\left(S_{0} \times\left\{i_{1}\right\}\right) \cup\left(\left\{i_{0}\right\} \times S_{1}\right),\left(i_{0}, i_{1}\right), L_{0} \cup L_{1}, \operatorname{tran}^{\prime}\right)
$$


where

$$
\left(\left(s, i_{1}\right), \alpha,\left(s^{\prime}, i_{1}\right)\right) \in \operatorname{tran}^{\prime} \text { iff }\left(s, \alpha, s^{\prime}\right) \in \operatorname{tran}_{0}
$$

and

$$
\left(\left(i_{0}, s\right), \alpha,\left(i_{0}, s^{\prime}\right)\right) \in \operatorname{tran}^{\prime} \text { iff }\left(s, \alpha, s^{\prime}\right) \in \operatorname{tran}_{1} .
$$

So, the sum construction is obtained by juxtaposing disjoint copies of the transition systems $T_{0}, T_{1}$ but identified at their initial states. The difference with Milner's sum are illustrated by this example:
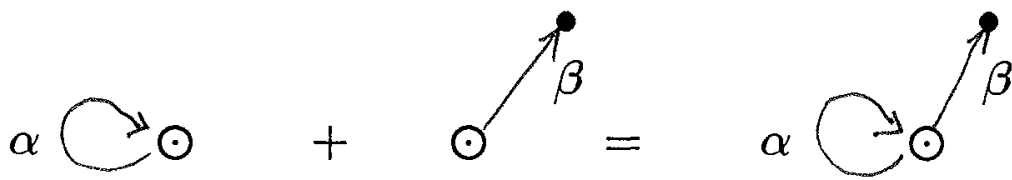

In the sum it is possible to arbitrarily many $\alpha$ transitions from one component and then do a $\beta$ transition; this is impossible for Milner's sum where once a transition occurs in one component of a sum then all future transitions must be from the same component. (The introduction of new states demanded by Milner's sum would complicate the reduction.)

A sum $T_{0}+T_{1}$ is associated with two injection functions on states:

$$
i n j_{i}: S_{i} \rightarrow\left(S_{0} \times\left\{i_{1}\right\}\right) \cup\left(\left\{i_{0}\right\} \times S_{1}\right), i=0,1
$$

with $i n j_{0}(s)=\left(s, i_{1}\right)$ and $i n j_{1}(s)=\left(i_{0}, s\right)$. They induce a map between properties:

$$
(-+-): P\left(T_{0}\right) \times P\left(T_{1}\right) \longrightarrow P\left(T_{0}+T_{1}\right)
$$

given by $V_{0}+V_{1}=\left\{i n j_{0}(s) \mid s \in V_{0}\right\} \cup\left\{i n j_{1}(s) \mid s \in V_{1}\right\}$ on $V_{0} \in P\left(T_{0}\right), V_{1} \in P\left(T_{1}\right)$.

Product: Let $T_{0}=\left(S_{0}, i_{0}, L_{0}, \operatorname{tran}_{0}\right)$ and $T_{1}=\left(S_{1}, i_{1}, L_{1}, \operatorname{tran}_{1}\right)$ be labelled transition systems. Their product $T_{0} \times T_{1}$ consists of states $S_{0} \times S_{1}$ with initial state $\left(i_{0}, i_{1}\right)$, labels $L_{0} \times_{*} L_{1}$ defined to be

$$
\left\{\left(\alpha_{0}, *\right) \mid \alpha \in L_{0}\right\} \cup\left\{\left(\alpha_{0}, \alpha_{1}\right) \mid \alpha_{0} \in L_{0}, \alpha_{1} \in L_{1}\right\} \cup\left\{\left(*, \alpha_{1}\right)\left|\alpha_{1}\right| \in L_{1}\right\},
$$

and transitions $\left(\left(s_{0}, s_{1}\right),\left(a_{0}, a_{1}\right),\left(s_{0}^{\prime}, s_{1}^{\prime}\right)\right)$ provided these satisfy:

$$
\begin{aligned}
& a_{0} \neq * \Rightarrow\left(s_{0}, a_{0}, s_{0}^{\prime}\right) \in \operatorname{tran}_{0} \text { and } a_{0}=* \Rightarrow s_{0}=s_{0}^{\prime}, \text { and } \\
& a_{1} \neq * \Rightarrow\left(s_{1}, a_{1}, s_{1}^{\prime}\right) \in \operatorname{tran}_{1} \text { and } a_{1}=* \Rightarrow s_{1}=s_{1}^{\prime} .
\end{aligned}
$$


Intuitively, the product allows arbitrary synchronisations between pairs of transitions in two components, allowing too for the possibility of a transition in one component proceeding independently of the other.

A product $T_{0} \times T_{1}$ is associated this map on properties:

$$
(-\times-): P\left(T_{0}\right) \times P\left(T_{1}\right) \rightarrow P\left(T_{0} \times T_{1}\right)
$$

where $V_{0} \times V_{1}$ is the cartesian product $\left\{\left(s_{0}, s_{1}\right) \mid s_{0} \in V_{0}, s_{1} \in V_{1}\right\}$.

Restriction: Let $T=(S, i, L, \operatorname{tran})$ be a labelled transition system. Given a subset of labels $\Lambda$ we can restrict the transitions of $T$ to those with labels in $\Lambda$. Define the restriction $T$ | $\Lambda=\left(S, i, L \cap \Lambda\right.$, tran $\left.^{\prime}\right)$ where $\operatorname{tran}^{\prime}=\left\{\left(s, \alpha, s^{\prime}\right) \in \operatorname{tran} \mid \alpha \in \Lambda\right\}$.

The reachable states of $T \vdash \Lambda$ are cut-down from those of $T$. There is an associated map on properties:

$$
(-\uparrow \Lambda): P(T) \longrightarrow P(T \uparrow \Lambda)
$$

where $V \uparrow \Lambda=\{s \in V \mid s$ reachable in $T \uparrow \Lambda\}$.

Relabelling: It is often useful to relabel the transitions of a labelled transition system. Let $T=(S, i, L$, tran $)$. Let $\Xi$ be a relabelling function from $L$ to labels. Define the relabelled transition system $T\{\Xi\}$ to be $\left(S, i, \Xi L, \operatorname{tran}^{\prime}\right)$ where where $\operatorname{tran}^{\prime}=\left\{(s, \Xi(\alpha), s) \mid\left(s, \alpha, s^{\prime}\right) \in\right.$ tran $\}$.

Relabelling leaves the states unaffected. Consequently any property of $T$ can be regarded as a property of $T\{\Xi\}$. Define

$$
(-\{\Xi\}) ; P(T) \longrightarrow P(T\{\Xi\})
$$

by taking $V\{\Xi\}=V$.

Looping: Let $T=(S, i, L, \operatorname{tran})$ be a labelled transition system. Assume $U$ denotes a property of $T$. Then by $T / \alpha, U$ we mean the transition system obtained from $T$ by introducing a transition $(s, \alpha, i)$ for each $s$ satisfying the property $U$. More concretely $T / \alpha, U$ is the $\left(S, i, L \cup\{\alpha\}, \operatorname{tran}^{\prime}\right)$ where $\operatorname{tran}^{\prime}=\operatorname{tran} \cup\{(s, \alpha, i) \mid s \in U\}$.

Like relabelling, the looping construct also leaves the states unaffected. Define

$$
(-/ \alpha, J): P(T) \rightarrow P(T / \alpha, J)
$$


by taking $(V / \alpha, J)=V$.

Parallel compositions: We can represent a variety of different parallel composition through a combined use of product, restriction and relabelling. For example, assuming a distinguished atomic label $\tau$ and a bijection $\alpha \mapsto \bar{\alpha}$ between non- $\tau$ atomic labels such that $\overline{\bar{\alpha}}=\alpha$, we can represent the parallel composition of CCS: take it to be

$$
T_{0} \mid T_{1}=_{\text {def }}\left(T_{0} \times T_{1} \mid \Lambda\right)\{\Xi\}
$$

where the restricting set $\Lambda$ consists of labels $(\alpha, *),(*, \alpha),(\alpha, \bar{\alpha}),(\tau, *),(*, \tau)$ where $\alpha$ ranges over all labels but for the distinguished label $\tau$, and the relabelling $\Xi$ acts so $\Xi(\alpha, *)=\Xi(*, \alpha)=\alpha$ and $\Xi(\tau, *)=\Xi(*, \tau)=$ $\Xi(\alpha, \bar{\alpha})=\tau$.

\section{$2 \quad$ Languages}

\subsection{Syntax}

Terms $t$ denote labelled transition systems with distinguished initial states. Assertions $A$ denote their properties. In fact an assertion $A$ will only sensibly denote a property of $t$ when a well-formedness judgement $A: t$ holds. The "raw" syntax of terms and assertions, ignoring for the moment their well-formedness, is mutually dependent and given as follows:

Terms:

$$
t::=n i l|\alpha t| t_{0}+t_{1}\left|t_{0} \times t_{1}\right| t|\Lambda| t\{\Xi\} \mid(t / \alpha, A)
$$

Assertions:

$$
\begin{aligned}
A:= & I|T| F\left|A_{0} \wedge A_{1}\right| \neg A \mid \\
& \langle a\rangle A|\overline{\langle a\rangle} A| \\
& X|\nu X . A| \\
& \alpha A\left|A_{0}+A_{1}\right| A_{0} \times A_{1}|A \vdash \Lambda| A\{\Xi\}\left|\left(A_{0} / \alpha, A_{1}\right)\right| \\
& (\vdash A: t)
\end{aligned}
$$

where $t$ is a term, $\alpha$ is a label, $\Lambda$ is a subset of labels, $\Xi$ is a relabelling function, $a$ is a label (possibly the 'idling' label $*$ ), and $X$ is an assertion 
variable. It is convenient to assume assertion variables belong to unique terms and we write $\operatorname{Var}(t)$ for the countably infinite set of assertion variables associated with the term $t$; so $\operatorname{Var}(t)$ and $\operatorname{Var}\left(t^{\prime}\right)$ are disjoint if $t$ and $t^{\prime}$ are distinct. Process terms $t$ are associated with a set of labels Labels $(t)$ defined by structural induction:

$$
\begin{aligned}
& \text { Labels }(n i l)=\emptyset, \quad \text { Labels }(\alpha t)=\text { Labels }(t) \cup\{\alpha\}, \\
& \text { Labels }\left(t_{0}+t_{1}\right)=\text { Labels }\left(t_{0}\right) \cup \text { Labels }\left(t_{1}\right), \\
& \text { Labels }\left(t_{0} \times t_{1}\right)=\text { Labels }\left(t_{0}\right) \times_{*} \text { Labels }\left(t_{1}\right), \\
& \text { Labels }(t \uparrow \Lambda)=\text { Labels }(t) \cap \Lambda, \quad \text { Labels }(t\{\Xi\})=\Xi \text { Label } s(t), \\
& \text { Labels }(t / \alpha, A)=\text { Labels }(t) \cup\{\alpha\} .
\end{aligned}
$$

We use Label $_{*}(t)$ to mean Labels $(t) \cup\{*\}$.

The assertion language is essentially a modal $\nu$-calculus with recursion. There are 'forwards' and 'backwards' modalities - the latter are useful in obtaining reductions for the product. The assertion $I$ will be used to refer to initial states; $I: t$ will denote the property holding just at the initial state of the transition system denoted by the process term $t$. In addition, assertions include constructions on properties with meanings described in the last section; these are used to build properties of a term from properties of its immediate components. It has another unusual construction: a validity assertion $(\vdash A: t)$ which will, in effect, be true or false according to whether or not $A$ is valid in $t$, with respect to a particular interpretation of its free variables as properties.

We shall employ some standard abbreviations, and write $A_{0} \wedge A_{1}$ for $\neg\left(\neg A_{0} \vee \neg A_{1}\right), A_{0} \rightarrow A_{1}$ for $\neg A_{0} \vee A_{1}, A_{0} \leftrightarrow A_{1}$ for $\left(A_{0} \rightarrow A_{1}\right) \wedge\left(A_{1} \rightarrow A_{0}\right)$. The minimum fixed point $\mu X$.A stands for $\neg \nu X . \neg A[\neg X / X]$. As regards substitution, we assume the usual renaming of bound variables to avoid the capture of free variables. In some reductions we use a nonstandard directed conditional

$$
B \rightarrow A_{0} \mid A_{1}
$$

to abbreviate $\left(B \wedge A_{0}\right) \vee A_{1}$. This is unusual; one would expect $(B \wedge$ $\left.A_{0}\right) \vee\left(\neg B \wedge A_{1}\right)$. The nonstandard choice is taken to avoid problems with monotonicity in the bodies of recursive definitions. Besides the directed conditional is always used in a context where the right arm $A_{1}$ is logically stronger than the left $A_{0}$; then the directed conditional $B \rightarrow A_{0} \mid A_{1}$ is logically equivalent to $\left(B \wedge A_{0}\right) \vee\left(\neg B \wedge A_{1}\right)$. 
The raw syntax allows assertions which are not sensible. For example, in the construct $\nu X . A$ care must be taken that the body $A$ determines a monotonic operator on sets of states. A sufficient condition for this is that all occurrences of the variable $X$ are positive, i.e. under an even number of negations; otherwise the recursive assertion is not well-formed. The judgement $A: t$ says when an assertion $A$ is well-formed as well as when it expresses a sensible property of a term $t$, once given properties for its free assertion variables. The well-formedness judgement is given by rules which are reminiscient of typing rules. This is consistent with the view that a process term is regarded as a type of properties. Well-formedness of assertions affects well-formedness of terms because the looping construct on terms $(t / \alpha, A)$ involves an assertion which we insist is closed and such that $A: t$.

Well-formedness rules:

$$
\begin{array}{llll}
I: t \quad T: t \quad F: t & \frac{A_{0}: t \quad A_{1}: t}{A_{0} \vee A_{1}: t} \quad \frac{A: t}{\neg A: t} \\
\frac{A: t \quad a \in \text { Label }_{*}(t)}{\langle a\rangle A: t} & \frac{A: t \quad a \in \text { Label }_{*}(t)}{\overline{\langle a\rangle} A: t} \\
X: t \quad \text { if } X \in \operatorname{Var}(t) & \frac{X: t \quad A: t \quad X+\text { ve in } A}{\nu X . A: t} \\
\frac{A: t}{\alpha A: \alpha t} & \frac{A_{0}: t_{0} \quad A_{1}: t_{1}}{A_{0}+A_{1}: t_{0}+t_{1}} & \frac{A_{0}: t_{0} \quad A_{1}: t_{1}}{A_{0} \times A_{1}: t_{0} \times t_{1}} \\
\frac{A: t}{A \uparrow \Lambda: t \uparrow \Lambda} & \frac{A: t}{A\{\Xi\}: t\{\Xi\}} & \frac{A_{0}: t \quad A_{1}: t \quad A_{1} \text { is closed }}{\left(A_{0} / \alpha, A_{1}\right): t / \alpha, A_{1}} \\
\frac{A: t_{0}}{\left(\vdash A: t_{0}\right): t} &
\end{array}
$$

Validity assertions, of the form $\vdash A: t$ will play a transient, though important, role in the reductions. Although the reductions will often introduce validity assertions, they can be removed so that subsequent reductions work on assertions free of them.

Definition: An assertion which does not contain any validity assertions will be called pure. 


\subsection{Semantics}

From the previous section, we understand each of the constructions in our language of process terms and so the denotation of a process term by a labelled transition system; in the case of the looping we will need to rely on the semantics of closed assertions as properties, made precise shortly.

Notation: In our subsequent work we will adopt the convention that a term $t$ denotes a labelled transition system

$$
\left(S_{t}, i_{t}, L_{t}, \operatorname{tran}_{t}\right)
$$

and write, for instance, $s \stackrel{\alpha}{\rightarrow} s_{t}^{\prime}$ to signify a transition in the transition system denoted by $t$. We shall write $P(t)$ for the set properties of (the transition system denoted by) $t$.

We give semantics to assertions $A$ accompanied by a judgement $A: t$. To cope with the possibility of free assertion variables in $A$, we use environments. Together assertion variables form the set

$$
\operatorname{Var}=\bigcup\{\operatorname{Var}(t) \mid t \in \operatorname{Term}\} .
$$

An environment $\rho$ is a function

$$
\rho: \operatorname{Var} \rightarrow \bigcup\{P(t) \mid t \in \operatorname{Term}\}
$$

such that $\rho(X) \in P(t)$ for $X \in \operatorname{Var}(t)$. Define Env to be the set of environments. The denotation of $A: t$ will be $\llbracket A: t \rrbracket$ of type Env $\rightarrow P(t)$. Define: 


$$
\begin{array}{lll}
\llbracket I: t \rrbracket & = & \lambda \rho \cdot\left\{i_{t}\right\} \\
\llbracket T: t \rrbracket & = & \lambda \rho \cdot S_{t} \\
\llbracket F: t \rrbracket & =\lambda \rho \cdot \emptyset \\
\llbracket A_{0} \vee A_{1}: t \rrbracket & =\lambda \rho \cdot \llbracket A_{0}: t \rrbracket \rho \cup \llbracket A_{1}: t \rrbracket \rho \\
\llbracket \neg A: t \rrbracket & =\lambda \rho \cdot\left(S_{t} \backslash \llbracket A: t \rrbracket \rho\right) \\
\llbracket\langle\alpha\rangle A: t \rrbracket & =\lambda \rho \cdot\left\{s \in S_{t} \mid \exists s^{\prime} . s \stackrel{\alpha}{\rightarrow}_{t} s^{\prime} \& s^{\prime} \in \llbracket A: t \rrbracket \rho\right\} \\
\llbracket X: t \rrbracket & =\lambda \rho \cdot \rho(X) \\
\llbracket \nu X \cdot A: t \rrbracket & =\lambda \rho \cdot(\nu U \cdot \llbracket A: t \rrbracket \rho[U / X]) \\
& & \text { the greatest fixed point of the function } \\
& & U \mapsto \llbracket A: t \rrbracket \rho[U / X] \\
\llbracket \alpha A: \alpha t \rrbracket & \lambda \rho \cdot \alpha(\llbracket A: t \rrbracket \rho) \\
\llbracket A_{0}+A_{1}: t_{0}+t_{1} \rrbracket & =\lambda \rho \cdot \llbracket A_{0}: t_{0} \rrbracket \rho+\llbracket A_{1}: t_{1} \rrbracket \rho \\
\llbracket A_{0} \times A_{1}: t_{0} \times t_{1} \rrbracket & =\lambda \rho \cdot \llbracket A_{0}: t_{0} \rrbracket \rho \times \llbracket A_{1}: t_{1} \rrbracket \rho \\
\llbracket A \vdash \Lambda: t \uparrow \Lambda \rrbracket & =\lambda \rho \cdot \llbracket A: t \rrbracket \rho\lceil\Lambda \\
\llbracket A\{\Xi\}: t\{\Xi\} \rrbracket & = & \lambda \rho \cdot(\llbracket A: t \rrbracket \rho)\{\Xi\} \\
\llbracket A_{0} / \alpha, A_{1}:\left(t / \alpha, A_{1}\right) \rrbracket= & \lambda \rho \cdot\left(\llbracket A_{0}: t \rrbracket \rho / \alpha, \llbracket A_{1} \rrbracket \rho\right) \\
\llbracket\left(\vdash A: t_{0}\right): t \rrbracket & =\lambda \rho \cdot\left(\llbracket A: t_{0} \rrbracket \rho=S_{t_{0}} \rightarrow S_{t} \mid \emptyset\right)
\end{array}
$$

Definition: (Validity) Let $A: t$ be an assertion. Define

$$
\vDash A: t \text { iff } \llbracket A: t \rrbracket \rho=S_{t} \text { for all environments } \rho .
$$

\section{How to do reductions}

We first motivate the technique by considering the reduction for the looping construct. Let $t$ be a term, $J: t$ a closed assertion. Then $(t / \alpha, J)$ denotes a transition system like that of $t$ but with extra $\alpha$ transitions from all the states satisfying $J$ to the initial state. Suppose $A$ is a closed assertion of the pure $\nu$-calculus (with no mention of validity assertions) so that $A:(t / \alpha, J)$. We describe how to produce an assertion $B: t$ such that

$$
\models A:(t / \alpha, J) \text { iff } \models B: t
$$

and in this way reduce the validity problem for a term $(t / \alpha, J)$ to one for $t$. The assertion $B$ will be defined by structural induction on $A$.

In the course of the structural induction we will generally encounter assertions which have free variables. To cope with this the reduction is 
done with respect to a substitution $\sigma$ transforming variables $X:(t / \alpha, J)$ to assertions $(Y / \alpha, J):(t / \alpha, J)$. In order not to introduce spurious dependencies it will be assumed that the free variables $Y$ do not appear free in any assertion being reduced and that $\sigma$ yields distinct $Y$ for distinct $X$. With respect to such a change of variables $\sigma$, we will consider a few clauses of the reduction, and indicate how it can be proved that if $A$ is pure with $A: t / \alpha, J$ then $\operatorname{red}(A: t / \alpha, J ; \sigma)=B$ with $B: t$ and

$$
\models A[\sigma] \leftrightarrow B / \alpha, J:(t / \alpha, J)
$$

This means that for all environments assigning properties to the free assertion variables, the assertions $A$ and $B / \alpha, J$ denote the same property of $t / \alpha, J$. It follows that, whenever $A: t / \alpha, J$ is closed,

$$
(\models A: t / \alpha, J) \text { iff }(\models B: t) \text {. }
$$

In this sense, a validity problem for a term $t / \alpha, J$ is reduced to one for $t$. We present a few clauses of the reduction:

$$
\begin{aligned}
\operatorname{red}(\langle\alpha\rangle A: t / \alpha, J ; \sigma)= & (\vdash I \rightarrow B: t) \rightarrow((\langle\alpha\rangle B) \vee J) \mid\langle\alpha\rangle B \\
& \text { where } \operatorname{red}(A: t / \alpha, J ; \sigma)=B \\
\operatorname{red}(X: t / \alpha, J ; \sigma)= & Y \text { when } \sigma(X)=Y / \alpha, J \\
\operatorname{red}(\nu X . A: t / \alpha, J ; \sigma)= & \nu Y \cdot \operatorname{red}(A: t / \alpha, J ; \sigma) \text { where } \sigma(X)=Y / \alpha, J .
\end{aligned}
$$

The second and third clauses express little more than a renaming of free variables. To understand the first reduction, assume inductively that

$$
\vDash A[\sigma] \leftrightarrow B / \alpha, J: t / \alpha, J
$$

and argue, for a state $s$ of $t / \alpha, J$ and arbitrary environment $\rho$, that

$$
\begin{aligned}
& s \in \llbracket\langle\alpha\rangle A[\sigma]:(t / \alpha, J) \rrbracket \rho \\
& \Longleftrightarrow \exists s^{\prime} . s \stackrel{\alpha}{\rightarrow} t / \alpha, J_{t} s^{\prime} \& s^{\prime} \in \llbracket A[\sigma]:(t / \alpha, J) \rrbracket \rho \\
& \Longleftrightarrow \exists s^{\prime} . s \stackrel{\alpha}{\rightarrow}_{t / \alpha, J} s^{\prime} \& s^{\prime} \in \llbracket B: t \rrbracket \rho \quad \text { by induction } \\
& \Longleftrightarrow \begin{cases}\left(\exists s^{\prime} . s \stackrel{\alpha}{\rightarrow}_{t} s^{\prime} \& s^{\prime} \in \llbracket B: t \rrbracket \rho\right) \text { or } s \in \llbracket J: t \rrbracket \rho & \text { if } i_{t} \in \llbracket B: t \rrbracket \rho, \\
\left(\exists s^{\prime} . s \stackrel{\alpha}{\rightarrow}_{t} s^{\prime} \& s^{\prime} \in \llbracket B: t \rrbracket \rho\right) & \text { if } i_{t} \notin \llbracket B: t \rrbracket \rho,\end{cases}
\end{aligned}
$$

directly from the looping construction,

$$
\begin{aligned}
& \Longleftrightarrow \begin{cases}s \in \llbracket(\langle\alpha\rangle B) \vee J: t \rrbracket \rho & \text { if } \llbracket \vdash I \rightarrow B: t \rrbracket \rho=S_{t} \\
s \in \llbracket\langle\alpha\rangle B: t \rrbracket \rho & \text { if } \llbracket \vdash I \rightarrow B: t \rrbracket \rho=\emptyset\end{cases} \\
& \Longleftrightarrow s \in \llbracket(\vdash I \rightarrow B: t) \rightarrow((\langle\alpha\rangle B) \vee J) \mid\langle\alpha\rangle B: t \rrbracket \rho
\end{aligned}
$$


There remains however one hitch. The reduction, like that for the other term constructors, works on pure assertions - those which do not contain validity assertions. As is clear from some of the clauses above, reductions can sometimes yield assertions with validity assertions. If we are now to continue the reduction (using the structure of $t$ ) we must show how to prepare such validity assertions so they can be handled by these further reductions.

Look at one clause where validity assertions are introduced:

$$
\operatorname{red}(\langle\alpha\rangle A: t / \alpha, J ; \sigma)=(\vdash I \rightarrow B: t) \rightarrow((\langle\alpha\rangle B) \vee J) \mid\langle\alpha\rangle B
$$

where $\operatorname{red}(A: t / \alpha, J ; \sigma)=B$. If $B$ is closed there are no difficulties: we check for the smaller term $t$ whether or not $I \rightarrow B$ is valid and if it is return the left, and otherwise the right branch of the conditional as the appropriate reduction. Validity assertions $\vdash B: t$ cause no difficulties when $B$ is closed. But in general $B$ will contain free assertion variables. However, ultimately we are concerned with reducing a closed assertion, which will mean that all free variables in validity assertions are bound by an enclosing recursive definition. The following fact means that, for a closed assertion denoting a property of $t$, the internal validity assertions introduced by its reduction can be made closed, and so benign because they refer to proper subterms of $t$ :

Lemma 1 (The closure lemma)

Let $\mathcal{C}[]$ be a context such that $\mathcal{C}[Y]: t$ and $Y$ occurs positively in $\mathcal{C}[Y]: t$, for any variable $Y: t_{0}$. Suppose $B: t_{0}$ and $\llbracket B: t_{0} \rrbracket \rho=\emptyset$ or $\llbracket B: t_{0} \rrbracket \rho=$ $S_{t_{0}}$, for any environment $\rho$. Let $X$ be a variable such that $\nu X . \mathcal{C}[B]: t$. Then

$$
\models \nu X . \mathcal{C}[B] \leftrightarrow \nu X . \mathcal{C}[B[\nu X . \mathcal{C}[T] / X]]: t
$$

As an illustration, consider the reduction of $\nu X .\langle\alpha\rangle X: t / \alpha, J$. This should yield an assertion of $t$ true at those reachable states of $t$ which become able to do arbitrarily many $\alpha$-transitions once the loops of the construction $t / \alpha, J$ are introduced. Assume the change of variables takes $X$ to $Y / \alpha, J$. Then, following the reductions above, we get

$$
\operatorname{red}(\nu X .\langle\alpha\rangle X: t / \alpha, J ; \sigma)=\nu Y .((\vdash I \rightarrow Y: t) \rightarrow((\langle\alpha\rangle Y) \vee J) \mid\langle\alpha\rangle Y) .
$$


By the closure lemma we can close the validity assertion, to obtain the equivalent

$$
\nu Y .(\vdash I \rightarrow \nu Y .((\langle\alpha\rangle Y) \vee J)): t) \rightarrow(\langle\alpha\rangle Y) \vee J \mid\langle\alpha\rangle Y .
$$

Thus $\operatorname{red}(\nu X .\langle\alpha\rangle X: t / \alpha, J ; \sigma)$ is equivalent to

1. $\nu Y .\langle\alpha\rangle Y$ if $\forall I \rightarrow \nu Y .((\langle\alpha\rangle Y) \vee J)$, and to

2. $\nu Y .((\langle\alpha\rangle Y) \vee J)$ if $\vdash I \rightarrow \nu Y .((\langle\alpha\rangle Y) \vee J)$.

In other words, a state in $t / \alpha, J$ can do arbitrarily many $\alpha$ transitions

1. if the corresponding state in $t$ can, or

2. it can reach a state in $J$ through $\alpha$-transitions and the initial state of $t$ can either do unboundedly many $\alpha$-transitions or itself reach a state in $J$ via $\alpha$-transitions.

This is the kind of result one could write down informally, except one might forget a case in 2. The informal argument is helped enormously through there being a simple reading of the recursive assertion. The reductions work for all manner of recursive assertions. I hope this indicates how the reductions perform rather complicated inference steps.

To illustrate more fully the issues involved in performing reductions we will consider the case of sums. Provided $A_{0}: t_{0}$ and $A_{1}: t_{1}$ then $A_{0}+A_{1}$ : $t_{0}+t_{1}$. This sum constructor on assertions reflects the operation we have seen for obtaining a property of a sum from properties of its components. For a closed assertion $A: t_{0}+t_{1}$ we are interested in how to produce assertions $A_{0}: t_{0}, A_{1}: t_{1}$ so that

$$
\models A \leftrightarrow A_{0}+A_{1}: t_{0}+t_{1} .
$$

Provided we can ensure in addition that the pair $A_{0}: t_{0}, A_{1}: t_{1}$ is balanced in the sense that

$$
i_{t_{0}} \in \llbracket A_{0}: t_{0} \rrbracket \rho \quad \text { iff } \quad i_{t_{1}} \in \llbracket A_{1}: t_{1} \rrbracket \rho,
$$

for all environments $\rho$, then

$$
\vDash A: t_{0}+t_{1} \quad \text { iff } \quad\left(\models A_{0}: t_{0} \text { and } \models A_{1}: t_{1}\right) .
$$


(Without the additional requirement of the assertions being balanced the "only if" direction of the statement can fail because only one of $A_{0}$ and $A_{1}$ can be true at the initial state.) The method for producing $A_{0}, A_{1}$ will work by induction on the structure of $A$, in the course of which we cannot hope to always deal with closed assertions. In particular, how are we to reduce a variable $X: t_{0}+t_{1}$ ? The answer rests on the fact that the reduction will take place relative to a change of variables, in which variables like $X$ are replaced by $Y_{0}+Y_{1}$ for distinct variables $Y_{0}: t_{0}$, $Y_{1}: t_{1}$.

To illustrate the mechanism of the reduction it is shown how, for a closed assertion $\nu X . A: t_{0}+t_{1}$, a balanced pair of assertions $B_{0}: t_{0}$ and $B_{1}: t_{1}$ can be found such that

$$
\vDash \nu X . A \leftrightarrow B_{0}+B_{1}: t_{0}+t_{1} .
$$

Of key importance are the maps between properties $P\left(t_{0}+t_{1}\right)$ and $P\left(t_{0}\right) \times$ $P\left(t_{1}\right)$. The change of variables is associated with the map

$$
\text { in }: P\left(t_{0}\right) \times P\left(t_{1}\right) \rightarrow P\left(t_{0}+t_{1}\right) \text { where } i n\left(V_{0}, V_{1}\right)=U_{0}+U_{1} .
$$

On the other hand, the reduction is associated with a map out in the converse direction

$$
\text { out }: P\left(t_{0}+t_{1}\right) \rightarrow P\left(t_{0}\right) \times P\left(t_{1}\right) \text { where out }(U)=\left(\text { out }_{0}(U), \text { out }_{1}(U)\right)
$$

which projects a property $U$ of $t_{0}+t_{1}$ to a pair of properties

$$
\begin{aligned}
& \text { out }_{0}(U)=\left\{s \in S_{t_{0}} \mid \text { inj }_{0}(s) \in U\right\}, \\
& \text { out }_{1}(U)=\left\{s \in S_{t_{1}} \mid \text { inj }_{1}(s) \in U\right\} .
\end{aligned}
$$

It is easy to see that the maps are monotonic with respect to inclusion and that out is anembedding of the properties $P\left(t_{0}+t_{1}\right)$ in $P\left(t_{0}\right) \times P\left(t_{1}\right)$ in the sense that in $\circ$ out $=1_{P\left(t_{0}+t_{1}\right)}$. These facts are important because they fit into a general pattern for transforming fixed points:

Lemma 2 (The embedding lemma)

Suppose $D$ and $E$ are complete lattices for which in $D \rightarrow E$ and out : $E \rightarrow D$ are monotonic, with in o out $=1_{E}$. Suppose $\phi: E \rightarrow E$ is monotonic. Defining

$$
\psi=o u t \circ \phi \circ i n
$$

we obtain a monotonic function $\psi: D \rightarrow D$ for which $\nu \phi=i n(\nu \psi)$. 
An assertion $A: t_{0}+t_{1}$ with a single free variable $X: t_{0}+t_{1}$ determines a function $\phi: P\left(t_{0}+t_{1}\right) \rightarrow P\left(t_{0}+t_{1}\right)$ from a property $U$ of $t_{0}+t_{1}$ to a property $\llbracket A: t_{0}+t_{1} \rrbracket \rho[U / X]$ of $t_{0}+t_{1}$. Suppose we have already obtained a reduction of $A$ to a balanced pair of assertions $A_{0}, A_{1}$ with respect to a change of variables taking $X$ to $Y_{0}+Y_{1}$, i.e.

$$
\models A\left[Y_{0}+Y_{1} / X\right] \leftrightarrow A_{0}+A_{1}: t_{0}+t_{1} .
$$

Then, equivalently, we can see the reduction as giving a syntactic expression of the embedding:

$$
\begin{aligned}
& \llbracket A_{0}: t_{0} \rrbracket \rho=\text { out }_{0}\left(\llbracket A\left[Y_{0}+Y_{1} / X\right]: t_{0}+t_{1} \rrbracket \rho\right) \\
& \llbracket A_{1}: t_{1} \rrbracket \rho=\text { out }_{1}\left(\llbracket A\left[Y_{0}+Y_{1} / X\right]: t_{0}+t_{1} \rrbracket \rho\right) .
\end{aligned}
$$

Writing $\psi_{0}\left(V_{0}, V_{1}\right)=\llbracket A_{0}: t_{0} \rrbracket \rho\left[V_{0} / Y_{0}, V_{1} / Y_{1}\right]$ and $\psi_{1}\left(V_{0}, V_{1}\right)=\llbracket A_{1}$ : $t_{1} \rrbracket \rho\left[V_{0} / Y_{0}, V_{1} / Y_{1}\right]$, yields

$$
\psi_{0}\left(V_{0}, V_{1}\right)=\operatorname{out}_{0}\left(\phi\left(V_{0}+V_{1}\right)\right) \text { and } \psi_{1}\left(V_{0}, V_{1}\right)=o u t_{1}\left(\phi\left(V_{0}+V_{1}\right)\right)
$$

for all $V_{0} \in P\left(t_{0}\right), V_{1} \in P\left(t_{1}\right)$. Now, defining $\psi$ by

$$
\psi\left(V_{0}, V_{1}\right)=\left(\psi_{0}\left(V_{0}, V_{1}\right), \psi_{1}\left(V_{0}, V_{1}\right)\right)
$$

for $V_{0} \in P\left(t_{0}\right), V_{1} \in P\left(t_{1}\right)$, we can see that $\psi=o u t \circ \phi \circ i n$. The wellformedness of assertions will ensure monotonicity of $\phi$ and $\psi$ so we can apply the embedding lemma to obtain:

$$
\nu U . \phi(U)=\operatorname{in}\left(\nu\left(V_{0}, V_{1}\right) \cdot\left(\psi_{0}\left(V_{0}, V_{1}\right), \psi_{1}\left(V_{0}, V_{1}\right)\right)\right) .
$$

By Bekic's theorem

$$
\nu U . \phi(U)=\operatorname{in}\left(\nu V_{0} . \psi_{0}\left(V_{0}, \nu V_{1} \cdot \psi_{1}\left(V_{0}, V_{1}\right)\right), \nu V_{1} \cdot \psi_{1}\left(\nu V_{0} . \psi_{0}\left(V_{0}, V_{1}\right), V_{1}\right)\right) .
$$

With an eye back to syntax, this means:

$$
\models \nu X . A \leftrightarrow\left(\nu Y_{0} \cdot A_{0}\left[\nu Y_{1} \cdot A_{1} / Y_{1}\right]+\nu Y_{0} \cdot A_{1}\left[\nu Y_{0} \cdot A_{0} / Y_{0}\right]\right): t_{0}+t_{1} .
$$

We have thus succeeded in producing a pair of assertions $B_{0}: t_{0}, B_{1}: t_{1}$ such that

$$
\models \nu X . A \leftrightarrow\left(B_{0}+B_{1}\right): t_{0}+t_{1} .
$$

A small additional argument, based on the assumption that $A_{0}: t_{0}, A_{1}: t_{1}$ are balanced, shows that $B_{0}: t_{0}, B_{1}: t_{1}$ form a balanced pair. Because 
$B_{0}: t_{0}, B_{1}: t_{1}$ denote fixed points of $\psi$ we see, for an arbitrary environment $\rho$, that

$$
\llbracket B_{0}: t_{0} \rrbracket \rho=\llbracket A_{0}: t_{0} \rrbracket \rho^{\prime} \text { and } \llbracket B_{1}: t_{1} \rrbracket \rho=\llbracket A_{1}: t_{1} \rrbracket \rho^{\prime}
$$

where $\rho^{\prime}=\rho\left[\llbracket B_{0}: t_{0} \rrbracket \rho / Y_{0}, \llbracket B_{1}: t_{1} \rrbracket \rho / Y_{1}\right]$. Now we observe that because the pair $A_{0}: t_{0}, A_{1}: t_{1}$ is balanced, so is $B_{0}: t_{0}, B_{1}: t_{1}$.

The reductions for the other constructions follow similar lines. Reductions will express embeddings of properties of a term in the properties, or products of properties, of its immediate components. They will be defined with respect to a change of variables associated with the left inverse to the embedding.

\section{Summary of results}

We now describe how to perform reductions for all the operations. As with the reduction for the looping construct, we shall need to change variables, so as to transform properties of a term to corresponding properties of its immediate subcomponents. We shall call such transformations changes of variables. All such transformations will be achieved through substitutions which introduce only fresh variables over properties.

Definition: A substitution $\sigma$ is said to be fresh for an assertion $A$ if it has the properties:

(i) for all variables $X$ at which $\sigma$ is defined the free variables in $\sigma(X)$ are disjoint from those in $A$, and

(ii) for distinct variables $X$ and $X^{\prime}$, at which $\sigma$ is defined, the free variables in $\sigma(X)$ and $\sigma\left(X^{\prime}\right)$ are disjoint.

Many of the reductions will introduce validity assertions. These are harmless however. They will always be validity assertions with respect to a smaller term than that of immediate interest, and, through the use of the closure lemma, lemma 1, they can be made closed whenever they arise as the reduction of a closed assertion; as such they can be checked, replaced by $T$ or $F$ as appropriate, and hence eliminated. 


\subsection{The reduction for nil}

Given a closed, pure assertion $A: n i l$, it is a simple matter to see whether or not it is valid at nil. The following function yields true in case it is valid, and false otherwise:

$$
\begin{array}{ll}
\operatorname{red}(I: n i l) & =\text { true } \\
\operatorname{red}(T: n i l) & =\text { true } \\
\operatorname{red}(F: n i l) & =\text { false } \\
\operatorname{red}\left(A_{0} \vee A_{1}: n i l\right) & =\operatorname{red}\left(A_{0}: n i l\right) \text { or }\left(\operatorname{red}\left(A_{1}: n i l\right)\right. \\
\operatorname{red}(\neg A: n i l) & =\operatorname{not} \operatorname{red}(A: n i l) \\
\operatorname{red}(\langle *\rangle: n i l) & =\operatorname{red}(A: n i l) \\
\operatorname{red}(\overline{\langle *} A: n i l) & =\operatorname{red}(A: n i l) \\
\operatorname{red}(\nu X . A: n i l) & =\operatorname{red}(A[T / X]: n i l) .
\end{array}
$$

Theorem 3 For $A$ : nil a closed, pure assertion, $\operatorname{red}(A:$ nil $)$ iff $\models A$ : nil.

\subsection{Reduction for prefixing}

The reduction for prefixing is based on an embedding of $P(\alpha t)$ into $P(t) \times$ $P(t)$, for a term $t$, meeting the requirements of the embedding lemma 2 . Define down $: P(\alpha t) \rightarrow P(t)$ by taking

$$
\operatorname{down}(U)=\left\{s \in S_{t} \mid\{s\} \in U\right\} .
$$

Define cont $I: P(\alpha t) \rightarrow P(t)$ by taking

$$
\operatorname{cont} I(U)=\left\{i_{t} \mid i_{\alpha t} \in U\right\} .
$$

Now we take the embedding to be

$$
\text { out }=(\text { down, cont } I): P(\alpha t) \rightarrow P(t)^{2}
$$

so that $\operatorname{out}(U)=(\operatorname{down}(U)$, cont $I(U))$. The converse map arises by taking

$$
\text { in }: P(t)^{2} \rightarrow P(\alpha t)
$$

where

$$
i n\left(V_{0}, V_{1}\right)=\alpha\left(V_{0}\right) \cup\left\{i_{\alpha t} \mid i_{t} \in V_{1}\right\}
$$


It is easy to check that both $i n$ and out are monotonic and inoout $=1_{P(\alpha t)}$. The map in corresponds to a change of variables, with respect to which we'll define a reduction whose two components correspond to the two components of the embedding out.

Definition: Assume $A: \alpha t$. A change of variables of $A: \alpha t$ is a substitution $\sigma$, with domain $\operatorname{Var}(\alpha t)$, which is fresh for $A$, and such that for all variables $X: \alpha t$ there are distinct variables $Y_{0}: t$ and $Y_{1}: t$ with

$$
\sigma(X)=\alpha\left(Y_{0}\right) \vee\left(\left(\vdash I \rightarrow Y_{1}: t\right) \wedge I\right)
$$

This change of variables expresses the map in:

Proposition 4 Let $X: \alpha t, Y_{0}: t$ and $Y_{1}: t$ be distinct variables. Suppose

$$
\sigma(X)=\alpha\left(Y_{0}\right) \vee\left(\left(\vdash I \rightarrow Y_{1}: t\right) \wedge I\right) .
$$

Let $\rho$ be an environment. Then, for $V_{0}, V_{1} \in P(t)$

$$
\operatorname{in}\left(V_{0}, V_{1}\right)=\llbracket \sigma(X): \alpha t \rrbracket \rho\left[V_{0} / Y_{0}, V_{1} / Y_{1}\right]
$$

Given a pure assertion $A: \alpha t$ and $\sigma$ a change of variables for it we define two functions

$\operatorname{red}^{0}(A: \alpha t ; \sigma)$ and $\operatorname{red}^{1}(A: \alpha t ; \sigma)$, such that

$$
\begin{array}{r}
\llbracket r e d^{0}(A: \alpha t ; \sigma): t \rrbracket \rho=\operatorname{down}(\llbracket A[\sigma]: \alpha t \rrbracket \rho) \\
\llbracket r e d^{1}(A: \alpha t ; \sigma): t \rrbracket \rho \cap\left\{i_{t}\right\}=\operatorname{cont} I(\llbracket A[\sigma]: \alpha t \rrbracket \rho)
\end{array}
$$

for any environment $\rho$.

By structural induction on $A: \alpha t$, with respect to a change of variables for it, define 


$$
\begin{aligned}
& \operatorname{red}^{0}(I: \alpha t ; \sigma) \quad=F \\
& \operatorname{red}^{0}(T: \alpha t ; \sigma) \quad=T \\
& \operatorname{red}^{0}(F: \alpha t ; \sigma) \quad=F \\
& \operatorname{red}^{0}\left(A_{0} \vee A_{1}: \alpha t ; \sigma\right)=\operatorname{red}^{0}\left(A_{0}: \alpha t ; \sigma\right) \vee \operatorname{red}^{0}\left(A_{1}: \alpha t ; \sigma\right) \\
& \operatorname{red}^{0}(\neg A: \alpha t ; \sigma) \quad=\neg r e d^{0}(A: \alpha t ; \sigma) \\
& \operatorname{red}^{0}(\langle a\rangle A: \alpha t ; \sigma) \quad=\langle a\rangle \operatorname{red}^{0}(A: \alpha t, \sigma) \\
& \operatorname{red}^{0}(\overline{\langle a\rangle} A: \alpha t ; \sigma) \quad=\overline{\langle a\rangle} r e d^{0}(A: \alpha t ; \sigma) \text { if } a \neq \alpha \\
& \operatorname{red}^{0}(\overline{\langle\alpha\rangle} A: \alpha t ; \sigma)=\overline{\langle\alpha\rangle} \operatorname{red}^{0}(A: \alpha t ; \sigma) \vee\left(\operatorname{red}^{1}(A: \alpha t ; \sigma) \wedge I\right) \\
& \operatorname{red}^{0}(X: \alpha t ; \sigma) \quad=Y_{0} \text { where } \\
& \sigma(X)=\alpha\left(Y_{0}\right) \vee\left(\left(\vdash I \rightarrow Y_{1}: t\right) \wedge I .\right) \\
& \left.\operatorname{red}^{0}(\nu X . A: \alpha t ; \sigma) \quad=\nu Y_{0} \cdot \operatorname{red}^{0}(A: \alpha t ; \sigma)\left[\nu Y_{1} \cdot \operatorname{red}^{1}(A: \alpha t ; \sigma) / Y_{1}\right]\right) \text { where } \\
& \sigma(X)=\alpha\left(Y_{0}\right) \vee\left(\left(\vdash I \rightarrow Y_{1}: t\right) \wedge I\right) \\
& \operatorname{red}^{0}(\alpha A: \alpha t ; \sigma) \quad=A \\
& \operatorname{red}^{1}(I: \alpha t ; \sigma) \quad=T \\
& \operatorname{red}^{1}(T: \alpha t ; \sigma) \quad=T \\
& \operatorname{red}^{1}(F: \alpha t ; \sigma) \quad=F \\
& \operatorname{red}^{1}\left(A_{0} \vee A_{1}: \alpha t ; \sigma\right)=\operatorname{red}^{1}\left(A_{0}: \alpha t ; \sigma\right) \vee \operatorname{red}^{1}\left(A_{1}: \alpha t ; \sigma\right) \\
& \operatorname{red}^{1}(\neg A: \alpha t ; \sigma) \quad=\neg \operatorname{red}^{1}(A: \alpha t ; \sigma) \\
& \operatorname{red}^{1}(\langle *\rangle A: \alpha t ; \sigma) \quad=\operatorname{red}^{1}(A: \alpha t ; \sigma) \\
& \operatorname{red}^{1}(\langle a\rangle A: \alpha t ; \sigma) \quad=F \text { if } a \neq * \& a \neq \alpha \\
& \operatorname{red}^{1}(\langle\alpha\rangle A: \alpha t ; \sigma) \quad=\operatorname{red}^{0}(A: \alpha t ; \sigma) \\
& \operatorname{red}^{1}(\overline{\langle *\rangle} A: \alpha t ; \sigma) \quad=\operatorname{red}^{1}(A: \alpha t, \sigma) \\
& \operatorname{red}^{1}(\overline{\langle a\rangle} A: \alpha t ; \sigma) \quad=F \text { if } a \neq * \\
& \operatorname{red}^{1}(X: \alpha t ; \sigma) \quad=Y_{1} \text { where } \sigma(X)=\alpha\left(Y_{0}\right) \vee\left(\left(\vdash I \rightarrow Y_{1}: t\right) \wedge I\right) \\
& \operatorname{red}^{1}(\nu X . A: \alpha t ; \sigma)=\nu Y_{1} \cdot\left(\operatorname{red}^{1}(A: \alpha t ; \sigma)\left[\nu Y_{0} \cdot \operatorname{red}^{0}(A: \alpha t ; \sigma) / Y_{0}\right]\right) \\
& \operatorname{red}^{1}(\alpha A: \alpha t ; \sigma) \quad=F
\end{aligned}
$$

Theorem 5 Assume $A$ : at with $A$ pure. Suppose $\sigma$ is a change of variables of $A: \alpha t$. Let red $(A: \alpha t ; \sigma)=A_{0}$ and $\operatorname{red}^{1}(A: \alpha t ; \sigma)=A_{1}$. Then $A_{0}: t$ and $A_{1}: t$. Moreover

$$
\begin{array}{r}
\llbracket A_{0}: t \rrbracket \rho=\operatorname{down}(\llbracket A[\sigma]: \alpha t \rrbracket \rho) \text { and } \\
i_{t} \in \llbracket A_{1}: t \rrbracket \rho \quad \text { iff } \quad i_{\alpha t} \in \llbracket A[\sigma]: \alpha t \rrbracket \rho
\end{array}
$$

for any environment $\rho$.

If $A: \alpha t$ is closed then $(\models A: \alpha t)$ iff $\left(\models A_{0} \wedge\left(I \rightarrow A_{1}\right): t\right)$. 


\subsection{Reduction for sum}

The reduction will be based on an embedding of $P\left(t_{0}+t_{1}\right)$ in $P\left(t_{0}\right) \times P\left(t_{1}\right)$, for terms $t_{0}, t_{1}$. Define

$$
\begin{aligned}
& \text { out }_{0}: P\left(t_{0}+t_{1}\right) \rightarrow P\left(t_{0}\right), \\
& \text { out }_{1}: P\left(t_{0}+t_{1}\right) \rightarrow P\left(t_{1}\right)
\end{aligned}
$$

by taking out $t_{0}(U)=\left\{s \in S_{t_{0}} \mid\right.$ inj $\left._{0}(s) \in U\right\}$ and out $_{1}(U)=\left\{s \in S_{t_{1}} \mid\right.$ $\left.i n j_{1}(s) \in U\right\}$ for all $U \in P\left(t_{0}+t_{1}\right)$. Define the embedding

$$
\text { out : } P\left(t_{0}+t_{1}\right) \rightarrow P\left(t_{0}\right) \times P\left(t_{1}\right)
$$

by taking out $(U)=\left(\right.$ out $_{0}(U)$, out $\left._{1}(U)\right)$ for all $U \in P\left(t_{0}+t_{1}\right)$. Define its left-inverse

$$
\text { in }: P\left(t_{0}\right) \times P\left(t_{1}\right) \rightarrow P\left(t_{0}+t_{1}\right)
$$

by taking in $\left(V_{0}, V_{1}\right)=V_{0}+V_{1}$. Both in and out are monotonic, and it is easily seen that in $\circ$ out $=1_{P\left(t_{0}+t_{1}\right)}$.

The map in accompanies a change of variables:

Definition: Let $A: t_{0}+t_{1}$. A change of variables of $A: t_{0}+t_{1}$ is a substitution $\sigma$ with domain $\operatorname{Var}\left(t_{0}+t_{1}\right)$, which is fresh for $A$, and such that for any variable $X: t_{0}+t_{1}$ we have $\sigma(X)=Y_{0}+Y_{1}$ for distinct variables $Y_{0}: t_{0}$ and $Y_{1}: t_{1}$.

Proposition 6 Let $X: t_{0}+t_{1}, Y_{0}: t_{0}$ and $Y_{1}: t_{1}$ be distinct variables. Suppose $\sigma(X)=Y_{0}+Y_{1}$. Let $\rho$ be an environment. Then, for $V_{0} \in P\left(t_{0}\right)$, $V_{1} \in P\left(t_{1}\right)$

$$
\operatorname{in}\left(V_{0}, V_{1}\right)=\llbracket \sigma(X): \alpha t \rrbracket \rho\left[V_{0} / Y_{0}, V_{1} / Y_{1}\right]
$$

With respect to a change of variables $\sigma$, we can transform an assertion $A: t_{0}+t_{1}$ to the sum of a pair of assertions $A_{0}: t_{0}$ and $A_{1}: t_{1}$ which realise the components of the embedding out, i.e.

$\llbracket A_{0}: t_{0} \rrbracket \rho=$ out $_{0}\left(\llbracket A[\sigma]: t_{0}+t_{1} \rrbracket \rho\right) \quad$ and $\quad \llbracket A_{1}: t_{1} \rrbracket \rho=o u t_{1}\left(\llbracket A[\sigma]: t_{0}+t_{1} \rrbracket \rho\right)$

for any environment $\rho$. 
The reduction is carried out by the pair of functions $\operatorname{red}^{0}\left(A: t_{0}+t_{1} ; \sigma\right)$, $\operatorname{red}^{1}\left(A: t_{0}+t_{1} ; \sigma\right)$, acting on an assertion $A$ for which $A: t_{0}+t_{1}$ and a change of variables for it. They are defined by the following structural induction (we omit the clauses for $r e d^{1}$ as they reflect those for $r e d^{0}$ ):

$$
\begin{aligned}
& \operatorname{red}^{0}\left(I: t_{0}+t_{1} ; \sigma\right) \quad=I \\
& \operatorname{red}^{0}\left(T: t_{0}+t_{1} ; \sigma\right) \quad=T \\
& \operatorname{red}^{0}\left(F: t_{0}+t_{1} ; \sigma\right) \quad=F \\
& \operatorname{red}^{0}\left(A \vee B: t_{0}+t_{1} ; \sigma\right) \quad=A_{0} \vee B_{0} \\
& \text { where } A_{0}=\operatorname{red}^{0}\left(A: t_{0}+t_{1} ; \sigma\right) \text { and } \\
& B_{0}=\operatorname{red}^{0}\left(B: t_{0}+t_{1} ; \sigma\right) \\
& \operatorname{red}^{0}\left(\neg A: t_{0}+t_{1} ; \sigma\right) \quad=\neg r e d^{0}\left(A: t_{0}+t_{1} ; \sigma\right) \\
& \operatorname{red}^{0}\left(\langle *\rangle A: t_{0}+t_{1} ; \sigma\right) \quad=\operatorname{red}^{0}\left(A: t_{0}+t_{1} ; \sigma\right) \\
& \operatorname{red}^{0}\left(\langle\alpha\rangle A: t_{0}+t_{1} ; \sigma\right) \quad=\left(\vdash I \rightarrow\langle\alpha\rangle A_{1}: t_{1}\right) \rightarrow\left(\langle\alpha\rangle A_{0}\right) \vee I \mid\langle\alpha\rangle A_{0} \text { where } \\
& \operatorname{red}^{0}\left(A: t_{0}+t_{1} ; \sigma\right)=A_{0} \text { and } \\
& \operatorname{red}^{1}\left(A: t_{0}+t_{1} ; \sigma\right)=A_{1} \text {, } \\
& \text { if } \alpha \neq * \\
& \operatorname{red}^{0}\left(\overline{\langle *\rangle} A: t_{0}+t_{1} ; \sigma\right) \quad=\operatorname{red}^{0}\left(A: \underline{\left.t_{0}+t_{1} ; \sigma\right)}\right. \\
& \left.\operatorname{red}^{0} \overline{\langle\alpha\rangle} A: t_{0}+t_{1} ; \sigma\right)=\left(\left(\vdash I \rightarrow \overline{\langle\alpha\rangle} A_{1}: t_{1}\right) \rightarrow\left(\overline{\langle\alpha\rangle} A_{0}\right) \vee I \mid \overline{\langle\alpha\rangle} A_{0}\right. \text { where } \\
& \operatorname{red}^{0}\left(A: t_{0}+t_{1} ; \sigma\right)=A_{0} \text { and } \\
& \operatorname{red}^{1}\left(A: t_{0}+t_{1} ; \sigma\right)=A_{1} \text {, } \\
& \text { if } \alpha \neq * \\
& \operatorname{red}^{0}\left(X: t_{0}+t_{1} ; \sigma\right) \quad=\left(\vdash I \rightarrow Y_{1}: t_{1}\right) \rightarrow Y_{0} \vee I \mid Y_{0} \quad \text { where } \sigma(X)=Y_{0}+Y_{1} \\
& \operatorname{red}^{0}\left(\nu X . A: t_{0}+t_{1} ; \sigma\right)=\nu Y_{0} \cdot A_{0}\left[\nu Y_{1} \cdot A_{1} / Y_{1}\right] \text { where } \\
& A_{0}=\operatorname{red}^{0}\left(A: t_{0}+t_{1} ; \sigma\right) \\
& A_{1}=\operatorname{red}^{1}\left(A: t_{0}+t_{1} ; \sigma\right) \\
& \text { and } \sigma(X)=Y_{0}+Y_{1} \\
& \operatorname{red}^{0}\left(A_{0}+A_{1}: t_{0}+t_{1} ; \sigma\right)=\left(\vdash I \rightarrow A_{1}: t_{1}\right) \rightarrow\left(A_{0} \vee I\right) \mid A_{0}
\end{aligned}
$$

Theorem 7 Let $A: t_{0}+t_{1}$ be pure. Let $A_{0}=\operatorname{red}^{0}\left(A: t_{0}+t_{1} ; \sigma\right)$ and $A_{1}=\operatorname{red}^{1}\left(A: t_{0}+t_{1} ; \sigma\right)$, for $\sigma$ a change of variables for $A$. Then

$$
\llbracket A_{0}: t_{0} \rrbracket \rho=\text { out }_{0}\left(\llbracket A[\sigma]: t_{0}+t_{1} \rrbracket \rho\right) \quad \text { and } \quad \llbracket A_{1}: t_{1} \rrbracket \rho=\text { out }_{1}\left(\llbracket A[\sigma]: t_{0}+t_{1} \rrbracket \rho\right)
$$

for any environment $\rho$.

If $A: t_{0}+t_{1}$ is closed then $\left(\models A: t_{0}+t_{1}\right)$ iff $\left[\left(\models A_{0}: t_{0}\right)\right.$ and $\left.\left(\models A_{1}: t_{1}\right)\right]$. 


\subsection{Reduction for looping.}

The properties $P(t / \alpha, J)$ and $P(t)$ are the same, and this time the embedding and its inverse with respect to which the reduction is performed are both the identity map. The inverse is realised by a change of variables, which essentially just renames them:

Definition: Assume $A: t / \alpha, J$ with $A$ pure. A change of variables of $A: t / \alpha, J$ is a substitution $\sigma$, with domain $\operatorname{Var}(t / \alpha, J)$, which is fresh for $A$, and of the form $\sigma(X)=Y / \alpha, J$ for variables $X: t / \alpha, J$ and $Y: t$.

The effort goes into finding an assertion to an assertion $\operatorname{red}(A: t / \alpha, J ; \sigma)$, such that for a pure $A:(t / \alpha, J)$ and a change of variables $\sigma$ for it,

$$
\vDash A[\sigma] \leftrightarrow \operatorname{red}(A: t / \alpha, J ; \sigma): t / \alpha, J .
$$

The reduction of a pure assertion $A: t / \alpha, J$, with respect to a change of variables for it, is defined by structural induction:

$$
\begin{aligned}
& \operatorname{red}(I: t / \alpha, J ; \sigma) \quad=I \\
& \operatorname{red}(T: t / \alpha, J ; \sigma) \quad=T \\
& \operatorname{red}(F: t / \alpha, J ; \sigma) \quad=F \\
& \operatorname{red}\left(A_{0} \vee A_{1}: t / \alpha, J ; \sigma\right)=\operatorname{red}\left(A_{0}: t / \alpha, J ; \sigma\right) \vee \operatorname{red}\left(A_{1}: t / \alpha, J ; \sigma\right) \\
& \operatorname{red}(\neg A: t / \alpha, J ; \sigma) \quad=\neg \operatorname{red}(A: t / \alpha, J ; \sigma) \\
& \operatorname{red}(\langle b\rangle A: t / \alpha, J ; \sigma)=\langle b\rangle \operatorname{red}(A: t / \alpha, J ; \sigma) \text { if } b \neq \alpha \\
& \operatorname{red}(\langle\alpha\rangle A: t / \alpha, J ; \sigma) \quad=(\vdash I \rightarrow B: t) \rightarrow((\langle\alpha\rangle B) \vee J) \mid\langle\alpha\rangle B \\
& \text { where } \operatorname{red}(A: t / \alpha, J ; \sigma)=B \\
& \operatorname{red}(\overline{\langle b\rangle} A: t / \alpha, J ; \sigma)=\overline{\langle b\rangle} \operatorname{red}(A: t / \alpha, J ; \sigma) \text { if } b \neq \alpha \\
& \operatorname{red}(\overline{\langle\alpha\rangle} A: t / \alpha, J ; \sigma) \quad=\neg(\vdash J \rightarrow \neg B: t) \rightarrow((\overline{\langle\alpha\rangle} B) \vee I) \mid \overline{\langle\alpha\rangle} B \\
& \text { where } \operatorname{red}(A: t / \alpha, J ; \sigma)=B \\
& \operatorname{red}(X: t / \alpha, J ; \sigma) \quad=Y \text { when } \sigma(X)=Y / \alpha, J \\
& \operatorname{red}(\nu X . A: t / \alpha, J ; \sigma)=\nu Y . r e d(A: t / \alpha, J ; \sigma) \text { where } \sigma(X)=Y / \alpha, J \\
& \operatorname{red}(A / \alpha, J: t / \alpha, J ; \sigma)=A \text {. }
\end{aligned}
$$

Theorem 8 Let $A$ be pure with $A: t / \alpha, J$. Let $\sigma$ be a change of variables of $A: t / \alpha, J$. Let $\operatorname{red}(A: t / \alpha, J ; \sigma)=B$. Then $B: t$ and moreover

$$
\vDash A[\sigma] \leftrightarrow B / \alpha, J: t / \alpha, J .
$$

If $A: t / \alpha, J$ is closed then $(\models A: t / \alpha, J)$ iff $(\models B: t)$. 


\subsection{Reduction for restriction}

Any property of a restriction $t \uparrow \Lambda$ can be regarded as a property of the component $t$; define

$$
\text { out }: P(t \uparrow \Lambda) \rightarrow P(t)
$$

by taking out $(U)=U$. The inverse map takes account of the fact that properties of $t$ | $\Lambda$ consist of states which are reachable via transitions within $\Lambda$. It is

$$
\text { in }: P(t) \rightarrow P(t \uparrow \Lambda)
$$

defined by $i n(V)=V \cap S_{i \uparrow \Lambda}$-note this means $i n(V)=V \uparrow \Lambda$. Both maps are monotonic and together satisfy in $\circ$ out $=1_{P(t, \Lambda)}$.

As usual the inverse map is associated with a change of variables:

Definition: Assume $A: t \uparrow \Lambda$. A change of variables of $A: t \uparrow \Lambda$ is a substitution $\sigma$, with domain $\operatorname{Var}(t \uparrow \Lambda)$, which is fresh for $A$, and of the form $\sigma(X)=Y \uparrow \Lambda$ for variables $X: t \uparrow \Lambda$ with $Y: t$ a variable.

With respect to a change of variables $\sigma$ we define a reduction of pure assertions $A: t \uparrow \Lambda$ to assertions $\operatorname{red}(A: t \uparrow \Lambda ; \sigma): t$ such that

$$
\models A[\sigma] \leftrightarrow \operatorname{red}(A: t \uparrow \Lambda ; \sigma) \uparrow \Lambda: t \uparrow \Lambda
$$

The reduction is related to the embedding out in the sense that

$$
\llbracket \operatorname{red}(A: t \uparrow \Lambda ; \sigma): t \rrbracket \rho \cap S_{t \uparrow \Lambda}=\operatorname{out}(\llbracket A[\sigma]: t \uparrow \Lambda \rrbracket \rho) .
$$

It is defined on pure assertion $A$ for which $A: t \uparrow \Lambda$ and reductions $\sigma$, on variables of them by the following structural induction: 


$$
\begin{aligned}
& \operatorname{red}(I: t \uparrow \Lambda ; \sigma) \quad=I \\
& \operatorname{red}(T: t, \Lambda ; \sigma)=T \\
& \operatorname{red}(F: t ; \Lambda ; \sigma) \quad=F \\
& \operatorname{red}\left(A_{0} \vee A_{1}: t \uparrow \Lambda ; \sigma\right)=\operatorname{red}\left(A_{0}: t \uparrow \Lambda, \sigma\right) \vee \operatorname{red}\left(A_{1}: t \uparrow \Lambda ; \sigma\right) \\
& \operatorname{red}(\neg A: t \mid \Lambda ; \sigma) \quad=\neg \operatorname{red}(A: t \mid \Lambda ; \sigma) \\
& \operatorname{red}(\langle\alpha\rangle A: t \uparrow \Lambda ; \sigma)= \begin{cases}F & \text { if } \alpha \notin \Lambda \\
\langle\alpha\rangle \operatorname{red}(A: t \uparrow \Lambda ; \sigma) & \text { if } \alpha \in \Lambda\end{cases} \\
& \operatorname{red}(\overline{\langle\alpha\rangle} A: t, \Lambda ; \sigma)= \begin{cases}\bar{F} & \text { if } \alpha \notin \Lambda \\
\overline{\langle\alpha\rangle}\left(R_{\Lambda} \wedge \operatorname{red}(A: t \uparrow \Lambda ; \sigma)\right) & \text { if } \alpha \in \Lambda\end{cases} \\
& \text { where } R_{\Lambda} \equiv \mu X . I \vee W_{\beta \in \Lambda}\langle\bar{\beta}\rangle X \\
& \operatorname{red}(X: t \uparrow \Lambda ; \sigma) \quad=Y \text { where } \sigma(X)=Y \vdash \Lambda \\
& \operatorname{red}(\nu X . A: t \uparrow \Lambda ; \sigma)=\nu Y . r e d(A: t \uparrow \Lambda ; \sigma) \text { where } \sigma(X)=Y \uparrow \Lambda \\
& \operatorname{red}(A \uparrow \Lambda: t \uparrow \Lambda ; \sigma) \quad=A \text {. }
\end{aligned}
$$

Theorem 9 Let $A$ be a pure assertion such that $A: t \mid \Lambda$, for which $\sigma$ is a change of variables. Let $\operatorname{red}\left(A: t{ }_{\uparrow} \Lambda ; \sigma\right)=B$. Then $B: t$ and

$$
\vDash A[\sigma] \leftrightarrow \operatorname{red}(A: t \uparrow \Lambda ; \sigma) \uparrow \Lambda: t \uparrow \Lambda
$$

If $A: t \uparrow \Lambda$ is closed then $(\models A: t \uparrow \Lambda)$ iff $\left(\models R_{\Lambda} \rightarrow B: t\right)$.

\subsection{Reduction for relabelling}

Because the properties $P(t)$ and $P(t\{\Xi\})$ are the same, the reductions and change of variables correspond to the identity map and are relatively straightforward.

Definition: Assume $A: t\{\Xi\}$. A change of variables of $A: t\{\Xi\}$ is a substitution $\sigma$, with domain $\operatorname{Var}(t\{\Xi\})$, which is fresh for $A$, and of the form $\sigma(X)=Y\{\Xi\}$ for variables $X: t\{\Xi\}$ and $Y: t$.

With respect to a change of variables $\sigma$, we define a reduction of a pure assertion $A: t\{\Xi\}$ to an assertion $\operatorname{red}(A: t\{\Xi\} ; \sigma)$ by structural induction: 


$$
\begin{aligned}
& \operatorname{red}(I: t\{\Xi\} ; \sigma) \quad=I \\
& \operatorname{red}(T: t\{\Xi\} ; \sigma) \quad=T \\
& \operatorname{red}(F: t\{\Xi\} ; \sigma) \quad=F \\
& \operatorname{red}\left(A_{0} \vee A_{1}: t\{\Xi\} ; \sigma\right)=\operatorname{red}\left(A_{0}: t\{\Xi\} ; \sigma\right) \vee \operatorname{red}\left(A_{1}: t\{\Xi\} ; \sigma\right) \\
& \operatorname{red}(\neg A: t\{\Xi\} ; \sigma) \quad=\quad \neg \operatorname{red}(A: t\{\Xi\} ; \sigma) \\
& \operatorname{red}(\langle *\rangle A: t\{\Xi\} ; \sigma) \quad=\operatorname{red}(A: t\{\Xi\} ; \sigma) \\
& \operatorname{red}(\langle\alpha\rangle A: t\{\Xi\} ; \sigma) \quad=W_{\beta \in \Xi^{-1}\{\alpha\}}\langle\beta\rangle \operatorname{red}(A: t\{\Xi\} ; \sigma) \\
& \operatorname{red}(\langle\bar{*}\rangle A: t\{\Xi\} ; \sigma) \quad=\operatorname{red}(A: t\{\Xi\} ; \sigma) \\
& \operatorname{red}(\langle\bar{\alpha}\rangle A: t\{\Xi\} ; \sigma) \quad=\mathrm{W}_{\beta \in \Xi^{-1}\{\alpha\}} \overline{\langle\beta\rangle} \operatorname{red}(A: t\{\Xi\} ; \sigma) \\
& \operatorname{red}(X: t\{\Xi\} ; \sigma) \quad=Y \text { where } \sigma(X)=Y\{\Xi\} \\
& \operatorname{red}(\nu X . A: t\{\Xi\} ; \sigma) \quad=\nu Y \cdot \operatorname{red}(A: t\{\Xi\} ; \sigma) \text { where } \sigma(X)=Y\{\Xi\} \\
& \operatorname{red}(A\{\Xi\}: t\{\Xi\} ; \sigma)=A \text {. }
\end{aligned}
$$

Theorem 10 Let $A$ be a pure assertion for such that $A: t\{\Xi\}$ for which $\sigma$ is a change of variables. Let $\operatorname{red}(A: t\{\Xi\} ; \sigma)=B$. Then $B: t$ and

$$
\models A[\sigma] \leftrightarrow B\{\Xi\}: t\{\Xi\}
$$

If $A: t\{\Xi\}$ is closed then $(\models A: t\{\Xi\})$ iff $(\models B: t)$.

\subsection{Reduction for product}

Looking back at the constructions so far, we see they share a common property, the presence of an embedding from properties of a constructed term to properties of its immediate components which are realised by the reductions on assertions of that term. Indeed, this reduction can be performed without looking at the composition of the immediate components; for instance, the reduction for $t_{0}+t_{1}$ proceeds independently of the composition of $t_{0}$ and $t_{1}$.

The difficulty in obtaining analogous reductions for parallel compositions stems from there not being such an embedding from properties of products to properties of their components. While there is the map

$$
(-\times-): P\left(t_{0}\right) \times P\left(t_{1}\right) \longrightarrow P\left(t_{0} \times t_{1}\right)
$$

There is no 1-1 map in the converse direction if one of $t_{0}, t_{1}$ has more than one and the other more than two reachable states-a little arithmetic 
shows that then the set $P\left(t_{0} \times t_{1}\right)$ has more states than $P\left(t_{0}\right) \times P\left(t_{1}\right)$. Reduction for assertions of a product, in general, cannot follow the same scheme as that of the other constructions. We are obliged to look for a different method of embedding and reduction, or at special kinds of properties in $P\left(t_{0} \times t_{1}\right)$ such as those which can embed in $P\left(t_{0}\right) \times P\left(t_{1}\right)$.

Properties having the shape $V_{0} \times V_{1}$, a cartesian product of $V_{0} \in P\left(t_{0}\right), V_{1} \in$ $P\left(t_{1}\right)$, are in correspondence with, and so embed in, properties $P\left(t_{0}\right) \times$ $P\left(t_{1}\right)$. By cutting down the properties of a product to those denoted by the following assertions, we can obtain a reduction:

$$
A::=I|T| B \times C|\langle(a, b)\rangle A| \overline{\langle(a, b)\rangle} A|((a, b)) A| \overline{((a, b))} A\left|A \wedge A^{\prime}\right| X \mid \nu X . A
$$

where we use $(a) A$ to abbreviate $[a] A \wedge\langle\alpha\rangle T$ and $\overline{(a)} A$ for $\overline{[a]} A \wedge \overline{\langle a\rangle} T$. Any closed assertion $A$ in this class, for which $A: t_{0} \times t_{1}$, has the property that

$$
\models A \leftrightarrow A_{0} \times A_{1}: t_{0} \times t_{1}
$$

for simply found $A_{0}: t_{0}, A_{1}: t_{1}$. These assertions for the components are obtained with respect to a change of variables for $t_{0} \times t_{1}$ which is a substitution, fresh for $A$, sending variables $X: t_{0} \times t_{1}$ to $Y_{0} \times Y_{1}$, for distinct variables $Y_{0}: t_{0}, Y_{1}: t_{1}$. Define:

$$
\begin{aligned}
\operatorname{red}\left(I: t_{0} \times t_{1} ; \sigma\right) & =(I, I) \\
\operatorname{red}\left(T: t_{0} \times t_{1} ; \sigma\right) & =(T, T) \\
\operatorname{red}\left(\langle(a, b)\rangle A: t_{0} \times t_{1} ; \sigma\right) & =\left(\langle a\rangle A_{0},\langle b\rangle A_{1}\right) \\
\operatorname{red}\left(\overline{\langle(a, b)\rangle} A: t_{0} \times t_{1} ; \sigma\right) & =\left(\overline{\langle a\rangle} A_{0}, \overline{\langle b\rangle} A_{1}\right) \\
\operatorname{red}\left(((a, b)) A: t_{0} \times t_{1} ; \sigma\right) & =\left((a) A_{0},(b) A_{1}\right) \\
\operatorname{red}\left(\overline{((a, b))} A: t_{0} \times t_{1} ; \sigma\right) & =\left(\overline{(a)} A_{0}, \overline{(b)} A_{1}\right) \text { where } \operatorname{red}\left(A: t_{0} \times t_{1} ; \sigma\right)=\left(A_{0}, A_{1}\right) \\
\operatorname{red}\left(A \wedge A^{\prime}: t_{0} \times t_{1} ; \sigma\right) & =\left(\left(A_{0} \wedge A_{0}^{\prime}\right),\left(A_{1} \wedge A_{1}^{\prime}\right)\right) \text { where } \\
\operatorname{red}\left(A: t_{0} \times t_{1} ; \sigma\right) & =\left(A_{0}, A_{1}\right), \operatorname{red}\left(A^{\prime}: t_{0} \times t_{1} ; \sigma\right)=\left(A_{0}^{\prime}, A_{1}^{\prime}\right) \\
\operatorname{red}\left(X: t_{0} \times t_{1} ; \sigma\right) & =\left(Y_{0}, Y_{1}\right) \text { where } \sigma(X)=Y_{0} \times Y_{1} \\
\operatorname{red}\left(\nu X . A: t_{0} \times t_{1} ; \sigma\right) & =\left(\left(\nu Y_{0} . A_{0}\right),\left(\nu Y_{1} . A_{1}\right)\right) \\
\text { where } \sigma(X) & =Y_{0} \times Y_{1} \text { and } \operatorname{red}\left(A: t_{0} \times t_{1} ; \sigma\right)=\left(A_{0}, A_{1}\right)
\end{aligned}
$$

While this reduction works for a nontrivial class of assertions the class is limited. In particular, it does not include the 'reachability' assertions $R_{\Lambda} \equiv \mu X . I \vee W_{\beta \in \Lambda}\langle\bar{\beta}\rangle X$, true of those states which are reachable from 
the initial state purely by transitions with labels in $\Lambda$. However, in the special case where

$$
\begin{aligned}
& \left(\lambda_{0}, \lambda_{1}\right) \in \Lambda \& \lambda_{0} \neq * \Rightarrow\left(\lambda_{0}, *\right) \in \Lambda, \text { and } \\
& \left(\lambda_{0}, \lambda_{1}\right) \in \Lambda \& \lambda_{1} \neq * \Rightarrow\left(*, \lambda_{1}\right) \in \Lambda
\end{aligned}
$$

we have that

$$
\models R_{\Lambda} \leftrightarrow R_{\Lambda_{0}} \times R_{\Lambda_{1}}: t_{0} \times t_{1}
$$

where $\Lambda_{0}=\left\{\lambda_{0} \mid \exists \lambda_{1} .\left(\lambda_{0}, \lambda_{1}\right) \in \Lambda\right\}, \Lambda_{1}=\left\{\lambda_{1} \mid \exists \lambda_{0} .\left(\lambda_{0}, \lambda_{1}\right) \in \Lambda\right\}$. As we shall see, this has implications for reductions with respect to the parallel composition of Milner's CCS.

Any recursion-free assertion of a product can be routinely transformed into a finite disjunction $W_{i \in I} B_{i} \times C_{i}$ (though conjunctions cause a quadratic 'blow-up' in size and negations an exponential 'blow-up'). As we have seen, there are special cases of recursive assertions where this can be achieved too. Once we have a property of a product $t_{0} \times t_{1}$ expressed in such a form, the following result provides a method for reducing its validity to validities in the components $t_{0}, t_{1}$. Note the result is independent of the composition of $t_{0}$ and $t_{1}$.

Proposition 11 Suppose $\mathrm{W}_{i \in I} B_{i} \times C_{i}: t_{0} \times t_{1}$ is a finite disjunction.

$$
\models W_{i \in I} B_{i} \times C_{i}: t_{0} \times t_{1}
$$

iff

$$
\left(\models W_{j \in J} B_{j}: t_{0}\right) \text { or }\left(\models W_{k \in K} C_{k}: t_{1}\right)
$$

for all partitions $J \cup K=I$.

It is useful to generalise the above proposition a little, so we can establish a property of a product relative to assumptions on the states of each component. This paper has concentrated on 'backwards proof'; given a goal for a compound term it has addressed how to reduce this to subgoals for its immediate components. One use of the following proposition is when asking the converse: if $\models B: t_{0}$ and $\models C: t_{1}$ does it follow that $\models A: t_{0} \times t_{1}$ ? The proposition provides a partial answer-it depends on $A$ having been expressed as a finite disjunction $\models W_{i \in I} B_{i} \times C_{i}: t_{0} \times t_{1}$. Then: 
Proposition 12 Suppose $\mathfrak{W}_{i \in I} B_{i} \times C_{i}: t_{0} \times t_{1}$ is a finite disjunction. Let $B: t_{0}, C: t_{1}$. Then

$$
\vDash B \times C \rightarrow W_{i \in I} B_{i} \times C_{i}: t_{0} \times t_{1}
$$

iff

$$
\left(\vDash B \rightarrow W_{j \in J} B_{j}: t_{0}\right) \text { or }\left(\models C \rightarrow W_{k \in K} C_{k}: t_{1}\right)
$$

for all partitions $J \cup K=I$.

As a corollary of this proposition, we obtain reduction results for certain parallel compositions, including that of CCS. A parallel composition of $t_{0}, t_{1}$ has the form

$$
t_{0} \| t_{1} \equiv\left(\left(t_{0} \times t_{1}\right) \uparrow \Lambda\right)\{\Xi\}
$$

A problem $\vDash A: t_{0} \| t_{1}$ reduces to

$$
\vDash R_{\Lambda} \rightarrow A^{\prime}: t_{0} \times t_{1}
$$

where $A^{\prime}$ is obtained by carrying out the reductions for relabelling, then restriction. (Note the reduction for restriction, as expressed by theorem 9 , introduces the reachability assertion $R_{\Lambda}$.) Now, in the case of $t_{0} \mid t_{1}$, for CCS, the appropriate restriction is with respect to a subset $\Lambda$ satisfying $(+)$. It follows that

$$
\vDash R_{\Lambda} \leftrightarrow R_{\Lambda_{0}} \times R_{\Lambda_{1}}: t_{0} \times t_{1}
$$

for reachability assertions $R_{\Lambda_{0}}$ and $R_{\Lambda_{1}}$. Hence if $A^{\prime}$ can be expressed as a manageable disjunction $W_{i \in I} B_{i} \times C_{i}$ we have a reductive way of checking $\models A: t_{0} \mid t_{1}$ :

$$
\models A: t_{0} \mid t_{1}
$$

iff

$$
\left(\vDash R_{\Lambda_{0}} \rightarrow W_{j \in J} B_{j}: t_{0}\right) \text { or }\left(\vDash R_{\Lambda_{1}} \rightarrow W_{k \in K} C_{k}: t_{1}\right)
$$

for all partitions $J \cup K=I$. Certainly, $A^{\prime}$ can be so decomposed when the original assertion $A$ contains no variables. Note that even for assertions $A$ without recursion, the statement $\models A: t_{0} \times t_{1}$, being one of validity, can express a nontrivial invariant of $t_{0} \times t_{1}$. It is emphasised that again this reduction does not depend on the structure of $t_{0}$ and $t_{1}$.

Unfortunately, the important use of restriction in CCS to internalise communication along a channel does not use a restricting set satisfying $(+)$. 
The problem with checking validity for terms which force internal communication along a channel centres on the difficulty of expressing

$$
R_{\{(a, b)\}} \equiv \mu X .(I \vee \overline{\langle(a, b)\rangle} X),
$$

true of those states in a product which are reachable from the initial state via a sequence of $(a, b)$-transitions, as a finite, and manageable, disjunction $W_{i \in I} B_{i} \times C_{i}: t_{0} \times t_{1}$. Of course, once we know the size of the transition system $t_{0} \times t_{1}$ to be $k$, we have

$$
\vDash R_{\{(a, b)\}} \leftrightarrow \mathbb{W}_{n<k} \overline{\langle a\rangle}^{n} I \times \overline{\langle b\rangle}^{n} I: t_{0} \times t_{1} .
$$

With luck, the recursion might become stationary at an earlier point, but to be a valid equivalence for all transition systems of size $k$ all the $k$ disjuncts have to be included. This reduction is thus quadratic in the size of the transition system. Certainly in this case the reduction can no longer be independent of $t_{0}, t_{1}$, with the assertion language as it stands presently.

\section{Conclusion}

General methods have been provided for reasoning compositionally with a modal $\nu$-calculus. These methods are presently being implemented by Henrik Andersen at Aarhus. Henrik has also extended the reductions to cope with a more traditional recursive definition of processes, which could be used in place of looping. The introduction of process variables which this entails could be useful for other reasons. Because all the reductions are directed only by the top-level operation on terms they might well be helpful in synthesising a process satisfying a specification, using process variables to leave parts of terms unspecified.

There remain important properties of products which do not seem directly amenable to the techniques outlined here. It is notable though that some nontrivial assertions have reductions which are independent of the structure of the components of a product. It is hoped that the techniques and limitations exposed here will help guide the search for methods of reasoning about parallel processes. Promising leads may be found in $[\mathrm{CLM}]$ and $[\mathrm{LX}]$. 
The approach here can be understood as running the compositional proof system of $[\mathrm{W}]$ backwards, and relates to the more modest compositional proof systems of [St] and [W1], and more superficially to [GS]. The reductions however have a fuller treatment of assertion variables than the proof system of [W]; the latter should be redone so that it supports the reasoning given by the reductions in a forwards direction and makes plain the sense in which the reductions correspond to running the proof system backwards.

\section{Acknowledgements}

I would like to acknowledge the support of the Esprit Basic Research Actions CEDISYS and CLICS. I've had valuable conversations with Henrik Andersen. Thanks to the referees for their comments. 


\section{References}

[CLM] Clarke, E., Long, D., and McMillan, K., Compositional model checking. LICS 1989.

[EL] Emerson, A. and Lei, C., Efficient model checking in fragments of the propositional mu-calculus. Proc.LICS, 1986.

[GS] Graf, I., and Sifakis, J., A logic for the description of nondeterministic programs and their properties. Report IMAG RR 551, Grenoble, France, 1985.

[LX] Larsen,K.G. and Xiuxin, L., Compositionality through an operational semantics of contexts. Proc. ICALP 90, 1990.

[St] Stirling, C, A complete modal proof system for a subset of SCCS. LNCS 185, 1985.

[W1] Winskel, G., A complete proof system for SCCS with modal assertions. In the proceedings of Foundations of Software Technology (1985).

[W] Winskel, G., A compositional proof system on a category of labelled transition systems. To appear in Information and Computation, 1990. 